Gastroenterologe 2018 $13: 444-449$ https://doi.org/10.1007/s11377-018-0304-3 Online publiziert: 25. Oktober 2018 (c) Der/die Autor(en) 2018 CrossMark

\author{
J.-M. Löhr' $\cdot$ T. Hackert ${ }^{2}$ \\ 'Gastrozentrum, Karolinska University Hospital und Karolinska Institutet, Stockholm, Schweden \\ ${ }^{2}$ Chirurgische Universitätsklinik, Universität Heidelberg, Heidelberg, Deutschland
}

\title{
Zystische Pankreasneoplasie - eine interdisziplinäre Herausforderung
}

der Seitengänge (Branched-duct[BD]IPMN) und den gemischten Formen (Mixed-type-IPMN) unterschieden. Die Prognose und der natürliche Verlauf sind sehr unterschiedlich (• Tab. 2).

\section{Derzeitige Herausforderungen}

Basierend auf den kürzlich veröffentlichten europäischen Richtlinien [7] lassen sich die folgenden Herausforderungen formulieren, auf die im Folgenden (• Tab. 3) unter Darstellung des derzeitigen Vorgehens eingegangen wird.

\section{Diagnostische Intervention}

Eine zufällig gefundene zystische Läsion wird mit einer State-of-the-artBildgebung, idealerweise MRT mit Magnetresonanzcholangiographie (MRCP) und Sekretinstimulation, bestmöglich dargestellt. Bei Unklarheiten hinsichtlich der Dignität werden weitere bildgebende Verfahren, wie 3-Phasen-CT und Endosonographie (EUS) mit Kontrastmittel, angewandt. Es erfolgt eine Einteilung entsprechend der WHO-Nomenklatur und im Fall von IPMN die Einteilung in Hauptgangläsionen (MDIPMN) oder Läsionen in den Seitengängen (BD-IPMN) sowie eine Beurteilung, ob sog. besorgniserregende Merkmale („worrisome features“), wie Größe $>3 \mathrm{~cm}$, Durchmesser des „main pancreatic duct“" (MPD) 5-9 mm, klinische Symptome, Pankreatitis, solide Knoten und/oder atypische Zellen in der Zytologie, vorliegen. „High-risk stigmata“ beinhalten Ikterus, MPD $\geq 10 \mathrm{~mm}$ sowie kontrastaufnehmende Knoten. Sollten danach noch
Unklarheiten existieren, ist eine kurzfristige (3-monatige) Kontrolluntersuchung mit Wiedervorstellung im Tumorboard erforderlich. Die Beurteilung der Konversion einer niedriggradigen Dysplasie in eine hochgradige ist mit derzeitigen bildgebenden Verfahren nicht möglich. In Einzelfällen kann die endoskopische retrograde Cholangiopankreatikographie (ERCP) mit Pankreatikoskopie [2] und gegebenenfalls sogar die konfokale Endomikroskopie (pCLE; [16]) hilfreich sein.

\section{》) Die Zytologie ist notorisch unzuverlässig}

Neben der Bildgebung gibt es weitere Kriterien, wie z. B. Tumormarker in Blut und Zystenflüssigkeiten sowie die Zytologie. Die Zytologie ist notorisch unzuverlässig mit $40 \%$ bis maximal $77 \%$, abhängig von der vermuteten Läsion [3]. Eine Messung der Pankreasenzyme in Serum und Zystenflüssigkeit ist bei der Fragestellung PCN nicht hilfreich, da bei Pseudozysten hohe Werte zu finden sind. Die Bestimmung des karzinoembryonalen Antigens (CEA) in der Zystenflüssigkeit ist vielleicht der etablierteste Parameter zur Differenzierung der muzinösen von den nichtmuzinösen Läsionen, auch wenn über den Cut-off-Wert noch keine Einigkeit herrscht [7, 23]. Der im Serum gemessene Tumormarker CA 199 scheint ebenfalls geeignet, zumindest bei den IPMN eine Invasion anzuzeigen: Wenn Werte im Verlauf, auch aus dem Normbereich (<37 I.E./l) heraus ansteigen, sollte man genau hinschauen. Es be- 
Tab. 1 Klinische, histopathologische und molekulare Charakteristika der häufigsten zystischen Neoplasien des Pankreas. (Nach [3])

\begin{tabular}{|c|c|c|c|c|}
\hline & IPMN & MCN & SCN & SPN \\
\hline Alter & $60-70$ & $40-50$ & $50-60$ & $30-40$ \\
\hline Geschlecht (m:w) & $60: 40$ & $5: 95$ & $40: 60$ & $20: 80$ \\
\hline Lokalisation & Caput (>80\%) & Cauda (>90\%) & Eher Cauda & Keine Präferenz \\
\hline Ganganschluss & Immer & Nein & Nein & Nein \\
\hline Häufigkeit (\%) & $20-25$ & $10-20$ & $<20$ & $<5$ \\
\hline Mutationsstatus & GNAS, KRAS & $\begin{array}{l}\text { KRAS, RNF43, SMAD4, Aneu- } \\
\text { ploidie }\end{array}$ & $V H L$, Aneuploidie & $\beta$-Catenin, PIK3CA \\
\hline Entartungsrate & Variabel & Häufig (>70\%) & Selten $(<5 \%)$ & Selten (10\%) \\
\hline Vorgehen & $\begin{array}{l}\text { Differenziert (Risikofakto- } \\
\text { ren!) } \\
\text { Bei Hauptgang-IPMN: } \\
\text { Resektion }\end{array}$ & Resektion & Überwachung & Resektion \\
\hline Besonderheiten & $\begin{array}{l}\text { Intestinaler, gastraler, } \\
\text { pankreatobiliärer, onko- } \\
\text { zytärer Subtyp }\end{array}$ & $\begin{array}{l}\text { Ovarähnliches Stroma, grö- } \\
\text { ßer als IPMN, hohes Maligni- } \\
\text { tätspotenzial }\end{array}$ & $\begin{array}{l}\text { Mikrozystisch/oligozystisch, Malignität } \\
\text { als Rarität }\end{array}$ & $\begin{array}{l}\text { „Junge Frauen“, un- } \\
\text { klare Pathogenese }\end{array}$ \\
\hline
\end{tabular}

IPMN intraduktale papillär-muzinöse Neoplasie, MCN muzinös-zystische Neoplasie, SCN serös-zystische Neoplasie, SPN serös-pseudopapilläre Neoplasie, $m$ männlich, $w$ weiblich

Tab. 2 Einteilung der intraduktalen papillär-muzinösen Neoplasien (IPMN). (Nach [4])

\begin{tabular}{|c|c|c|c|c|}
\hline & Gastrisch & Intestinal & Pankreatobiliär & Onkozytisch \\
\hline Lokalisation & Seitengang $>$ Hauptgang & Hauptgang $>$ Seitengang & Seitengang $>$ Hauptgang & Seitengang $>$ Hauptgang \\
\hline Wachstum & Flach & Villi & Komplex papillär & Solide \\
\hline Dysplasiegrad & LGD/IGD & IGD/HGD & HGD & HGD \\
\hline Marker & MUC5AC, MUC6 & MUC2, MUC5AC, CDX2, CK20 & MUC1, MUC5AC, MUC6 & MUC1 \\
\hline Pankreatitis & Häufig & Nein & Nein & Nein \\
\hline Häufigkeit (\%) & $20-25$ & $10-20$ & $<20$ & $<5$ \\
\hline Mutationsstatus & - & GNAS, KRAS & GNAS, KRAS & - \\
\hline $\begin{array}{l}\text { Vorliegen bei } \\
\text { invasivem Karzi- } \\
\text { nom (\%) }\end{array}$ & 15 & $30-60$ & $60-75$ & 25 \\
\hline $\begin{array}{l}\text { Art des invasiven } \\
\text { Karzinoms }\end{array}$ & $\begin{array}{l}\text { Konventionelles Adeno- } \\
\text { karzinom }\end{array}$ & Kolloid oder konventionell & $\begin{array}{l}\text { Konventionelles Adeno- } \\
\text { karzinom }\end{array}$ & $\begin{array}{l}\text { Onkozytisch oder } \\
\text { konventionell }\end{array}$ \\
\hline
\end{tabular}

darf allerdings weiterer großer prospektiver Studien mit exzellenter Bildgebung, um diesen Befund zu etablieren [7, 23].

\section{Chirurgische Intervention}

Die Entscheidung über Indikation und Zeitpunkt einer chirurgischen Intervention muss neben dem Typ der IPMN (BD, MD oder „mixed type“) immer individuelle Faktoren des betroffenen Patienten (v. a. Alter und Komorbidität) berücksichtigen. Hierbei werden grundsätzlich MD- und Mixed-type-IPMN als gemeinsame Entität betrachtet, da ihr Malignitätsrisiko vergleichbar hoch und von der Hauptgangkomponente abhängig ist [26]. In allen Leitlinien gilt diesbezüglich ein Gangdurchmesser von $\geq 10 \mathrm{~mm}$ als Operationsindikation im Sinne einer onkologischen Resektion (also partielle Duodenopankreatektomie [„Whipple-Operation“], Pankreaslinksresektion oder totale Duodenopankreatektomie inkl. entsprechender Lymphadenektomie), sofern der Patient sich von seinen Begleiterkrankungen für eine Operation qualifiziert [7, 27]. Da die Definition von MD- und Mixed-typeIPMN jedoch bereits einen Gangdurchmesser von $\geq 5 \mathrm{~mm}$ beinhaltet, stellt der Bereich zwischen 5 und $9,9 \mathrm{~mm}$ eine "Grauzone" dar, in der aktuell die Operationsindikation kontrovers diskutiert wird.
Während die Leitlinien der International Association of Pancreatology (IAP; [27]) hier lediglich ein „worrisome feature" sehen und somit keine Operation empfehlen, fehlt in der Leitlinie der American Gastroenterological Association (AGA; [28]) eine genaue Angabe des Hauptgangdurchmessers, sodass diese nicht für eine Entscheidung in diesem Bereich herangezogen werden kann. Im Gegensatz dazu wird in der aktualisierten europäischen Leitlinie [7] das Malignitätsrisiko auch bei einem Durchmesser zwischen 5 und 9,9 $\mathrm{mm}$ als hinreichend angesehen, um eine relative Operationsindikation zu empfehlen; d.h., bei vertretbarer Komorbidität sollten die betroffenen Patienten operiert werden, auch 
wenn keine weitere Risikokonstellation (z.B. erhöhtes CA 19-9) vorliegt. Hintergrund für diese Empfehlung sind verschiedene Arbeiten der vergangenen Jahre, in denen das Risiko für High-gradeDysplasien oder invasive Karzinome im Mittel bei $56 \%$ (30-91\%) lag [1, 9, 24]. Insbesondere vor dem Hintergrund, dass eine Resektion vor der Entwicklung eines invasiven Karzinoms durchgeführt werden sollte, erscheint diese Empfehlung sinnvoll.

Bei der BD-IPMN wurde in der Vergangenheit das Kriterium der Größe bei der Indikationsstellung zur Operation häufig stark in den Vordergrund gestellt. Daneben definierten die häufig angewandten sog. Sendai-FukuokaKonsensus-Leitlinien die bereits genannten „worrisome features“ und „high-risk stigmata“, anhand derer die Empfehlung zur weiteren Diagnostik bzw. Operation gegeben wurden. Inzwischen scheint sich abzuzeichnen, dass die Größe einer BD-IPMN alleine kein hinreichendes Kriterium für das Malignitätsrisiko und damit die Operationsindikation darstellt, da einerseits auch BD-IPMN unter $3 \mathrm{~cm}$ in verschiedenen Studien eine relevante Malignitätsrate aufwiesen $([8,10,11,13$, 22, 23, 29]; - Tab. 4) und andererseits auch große BD-IPMN häufig benigne sind, wenn keine anderen Kriterien für maligne Entartung vorliegen. In den aktuellen europäischen Leitlinien wurde daher die Größe als Kriterium bis $4 \mathrm{~cm}$ nicht mehr berücksichtigt, vielmehr stellen weitere radiologische und endosonographisch definierte Veränderungen wesentlich wichtigere Faktoren dar, auch die Wachstumsdynamik (unabhängig von der absoluten Größe) kann eine entscheidende Rolle spielen. Bei Vorliegen einer Wachstumsdynamik von $\geq 5 \mathrm{~mm} / \mathrm{Jahr}$, erhöhtem CA 19-9, neu aufgetretenem Diabetes mellitus, IPMN-assoziierter akuter Pankreatitis oder einem kontrastmittelaufnehmenden Knötchen $(<5 \mathrm{~mm})$ besteht damit unter $4 \mathrm{~cm}$ Größe eine relative Operationsindikation; d.h., ein sonst bezüglich Komorbiditäten gesunder Patient sollte operiert werden. Dies kann bei einer BD-IPMN nicht nur im Sinne einer onkologischen Resektion, die in der Regel möglich ist, sondern befundabhängig

Gastroenterologe 2018 $\cdot 13: 444-449$ https://doi.org/10.1007/s11377-018-0304-3

(c) Der/die Autor(en) 2018

\section{J.-M. Löhr $\cdot$ T. Hackert \\ Zystische Pankreasneoplasie - eine interdisziplinäre Herausforderung}

Zusammenfassung

Zystische Pankreasneoplasien (PCN) stellen eine zunehmend mehr Aufmerksamkeit erfordernde Gruppe von sehr verschiedenen Tumorentitäten dar, die aufgrund ihrer sehr unterschiedlichen Prognosen, basierend auf sehr divergierenden Malignitätspotenzialen, unterschiedlich behandelt werden müssen. Während einige obligate Präkanzerosen darstellen, müssen andere zunächst nur regelmäßig beobachtet werden und eine weitere Gruppe muss in der Regel nicht in ein Überwachungsprogramm aufgenommen werden. Diese unterschiedliche Biologie zeitigt diversifizierte diagnostische und therapeutische Aktionen, determiniert durch das Damokles-Schwert eines nicht rechtzeitig diagnostizierten und behandelten Pankreaskarzinoms.

Schlüsselwörter

Pankreasneoplasien · Präkanzeröse Läsion . Risikomanagement . Überwachungsprogram . Prognose

\section{Cystic pancreatic neoplasms-an interdisciplinary challenge}

\section{Abstract}

Cystic pancreatic neoplasms (PCNs) represent a group of very different types of tumors, which, due to their very different prognoses, have to be treated adequately based on their varying malignant potential. While some are obligatory precancerous lesions, others need to be monitored on a regular basis in a surveillance program and another group does not usually need to be included in such a surveillance program at all. This diverse biology results in diversified diagnostic and therapeutic actions, determined by the Damocles sword of a poorly diagnosed and treated pancreatic carcinoma that must be avoided.

\section{Keywords}

Pancreatic neoplasms - Precancerous conditions · Risk management · Population surveillance. Prognosis parenchymsparend (z. B. durch alleinige Enukleation des Befunde [12]) erfolgen.

》) Bei parenchymsparen-
der Operation muss ein
Schnellschnitt zum Malignitäts-
ausschluss erfolgen

Intraoperativ muss eine Malignität mittels Schnellschnitt ausgeschlossen werden, ansonsten muss die parenchymsparende Operation erweitert werden. Insbesondere bei BD-IPMN bieten sich außerdem minimalinvasive Verfahren (z.B. laparoskopische Enukleation oder Linksresektion) an, die bei entsprechender Expertise des Chirurgen auch robotisch assistiert erfolgen können [25]. Die potenziellen Vorteile dieser Verfahren sind neben kosmetisch besseren Ergebnissen eine geringeres Operati- onstrauma mit schnellerer Erholung und kürzerer Krankenhausverweildauer. Diese Vorteile sind bislang nicht durch methodologisch gute Studien (RCT) belegt. Entsprechende Studien werden daher zurzeit durchgeführt, sodass in den nächsten Jahren auch wissenschaftlich belegbare Ergebnisse hierzu vorliegen werden. Absolute Operationsindikationen bei BD-IPMN bestehen bei bioptisch nachgewiesener High-grade-Dysplasie oder bereits manifestem Karzinom sowie bei IPMN-bedingtem Ikterus und kontrastmittelaufnehmendem Knötchen $\geq 5 \mathrm{~mm}$. Auch Patienten mit relevanten Begleiterkrankungen sollten nach Abwägung des Operationsrisikos einer Resektion zugeführt werden.

In diesem Zusammenhang muss unterstrichen werden, dass gerade bei BDIPMN individuelle Entscheidungen für oder gegen eine Operation immer möglich sind, wobei v. a. ein hohes Lebensal- 
Hier steht eine Anzeige.

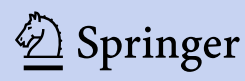


Tab. 3 Derzeitige Herausforderungen bei PCN

\begin{tabular}{|c|c|}
\hline Modalität & Problemstellung \\
\hline \multirow[t]{2}{*}{$\begin{array}{l}\text { Diagnostische } \\
\text { Intervention }\end{array}$} & $\begin{array}{l}\text { Welche Modalität ist am besten geeignet, neoplastische von nichtneoplas- } \\
\text { tischen Zysten zu unterscheiden? }\end{array}$ \\
\hline & $\begin{array}{l}\text { Welche Methode ist am zuverlässigsten in der Detektion hochgradiger } \\
\text { Dysplasien bei PCN, um eine rechtzeitige Resektion zu ermöglichen? }\end{array}$ \\
\hline \multirow[t]{4}{*}{ Chirurgie } & $\begin{array}{l}\text { Was sind absolute und relative Indikationen bei MD-IPMN und BD-IPMN, } \\
\text { speziell bei denen mit }>30 \mathrm{~mm} \text { Durchmesser? }\end{array}$ \\
\hline & $\begin{array}{l}\text { Wie ausgedehnt sollte bei MD-IPMN reseziert werden (partielle vs. totale } \\
\text { Pankreatektomie)? }\end{array}$ \\
\hline & $\begin{array}{l}\text { Gibt es eine Rolle für die prä- oder intraoperative Pankreatikoskopie } \\
\text { (SpyGlass }{ }^{\text {TM }} \text { DS [Boston Scientific Medizintechnik GmbH, Ratingen, Deutsch- } \\
\text { land])? }\end{array}$ \\
\hline & $\begin{array}{l}\text { Was ist der potentielle Benefit einer präventiven Chirurgie in Hinblick auf } \\
\text { mögliche Risiken und Nebenwirkungen der Chirurgie? }\end{array}$ \\
\hline \multirow[t]{3}{*}{$\begin{array}{l}\text { Überwachung } \\
\text { (Surveillance) }\end{array}$} & $\begin{array}{l}\text { Welches ist das kosteneffiziente und personalisierte Vorgehen bei einer } \\
\text { PCN? }\end{array}$ \\
\hline & $\begin{array}{l}\text { Was sind die relevanten Risikofaktoren für eine Progression (Zystengröße, } \\
\text { Rauchen, Diabetes mellitus, Familienanamnese für Krebserkrankungen, } \\
\text { genetische Syndrome)? }\end{array}$ \\
\hline & $\begin{array}{l}\text { Was sind das optimale bildgebende Verfahren und Follow-up-Schema nach } \\
\text { einer partiellen Resektion eines IPMN? }\end{array}$ \\
\hline
\end{tabular}

Tab. 4 Übersicht chirurgischer Studien zu Malignitätsraten bei intraduktaler papillär-muzinöser Neoplasie des Seitengangs $<30 \mathrm{~mm}$ und $>30 \mathrm{~mm}$

\begin{tabular}{|c|c|c|c|c|c|c|}
\hline \multirow[t]{2}{*}{ Studie } & \multirow[t]{2}{*}{$n$} & \multicolumn{5}{|c|}{ Malignitätsrate } \\
\hline & & $<10 \mathrm{~mm}(\%)$ & $\begin{array}{l}10-20 \mathrm{~mm} \\
(\%)\end{array}$ & $\begin{array}{l}20-30 \mathrm{~mm} \\
(\%)\end{array}$ & $\begin{array}{l}\text { Gesamt } \\
<30 \mathrm{~mm} \\
(\%)\end{array}$ & $\begin{array}{l}\text { Gesamt } \\
>30 \mathrm{~mm} \\
(\%)\end{array}$ \\
\hline $\begin{array}{l}\text { Schmidt et al. } \\
\text { [23] }\end{array}$ & 103 & $3 / 18(17)$ & $8 / 35(23)$ & $5 / 29(17)$ & $16 / 82(20)$ & 4/21 (19) \\
\hline Jang et al. [10] & 138 & $1 / 31(3)$ & $7 / 42(17)$ & $1 / 25(4)$ & $9 / 98(9)$ & $\begin{array}{l}12 / 40 \\
(30)\end{array}$ \\
\hline Fritz et al. [8] & 123 & $3 / 12(25)$ & $11 / 40(28)$ & $3 / 17(18)$ & $17 / 69(25)$ & $\begin{array}{l}16 / 54 \\
(30)\end{array}$ \\
\hline Wong et al. [29] & 105 & $4 / 7(57)$ & $5 / 19(26)$ & $31 / 44(70)$ & $40 / 70(57)$ & $\begin{array}{l}22 / 35 \\
(63)\end{array}$ \\
\hline $\begin{array}{l}\text { Sahora et al. } \\
\text { [22] }\end{array}$ & 217 & $0 / 4(0)$ & $6 / 46(13)$ & $15 / 75(20)$ & $21 / 125(17)$ & $\begin{array}{l}27 / 92 \\
(29)\end{array}$ \\
\hline Jang et al. [11] & 350 & - & - & - & $48 / 184(26)$ & $\begin{array}{l}49 / 166 \\
(30)\end{array}$ \\
\hline Kim et al. [13] & 324 & $0 / 3(0)$ & $3 / 60(5)$ & 10/111 (9) & $13 / 174(7)$ & $\begin{array}{l}29 / 150 \\
(19)\end{array}$ \\
\hline
\end{tabular}

ter (>70 Jahre), das Operationsrisiko, eine mögliche familiäre Belastung für das Auftreten von Pankreaskarzinomen und auch der Patientenwunsch berücksichtigt werden sollten.

\section{Surveillance}

Hier gilt es zunächst einmal festzustellen, ob es sich bei der relevanten Läsion um moren resektabel. Nach initialer Diagnosestellung, die ja in der Regel zufällig erfolgt, sollte die grundlegende Bildgebung im Rahmen eines Pankreas-MRT mit MRCP, wenn möglich unter Sekretinstimulation, erfolgen [18]. Die erste Kontrolle sollte, entsprechend den Leitlinien, nach 6 Monaten erfolgen, um eine bereits bestehende Wachstumstendenz nicht $\mathrm{zu}$ verpassen [7]. Offensichtlich sind unterschiedliche MR-Techniken wie auch die Messung von Diameter oder Volumen gut vergleichbar [20].

\section{》) Alle IPMN beinhalten ein lebenslanges Risiko für eine malige Entartung}

Zahlreichen Langzeitstudien haben gezeigt, dass alle IPMN ein lebenslanges Risiko für eine Wachstumsdynamik und eine malige Entartung beinhalten [5, 13]. Daher sollten Patienten mit dieser Diagnose in ein strukturiertes Überwachungsprogramm aufgenommen werden. Auch nach erfolgter Resektion einer IPMN muss ein lebenslanges Follow-up durchgeführt werden, es sei denn, es wurde eine totale Pankreatektomie ohne Nachweis eines invasiven Karzinoms durchgeführt. Ansonsten kann an jeder Stelle des Restpankreas nach Pankreasteilresektion erneut eine IPMN entstehen oder sich eine bestehende IPMN verändern. Die Empfehlungen sowohl der IAP wie auch der europäischen Leitlinien [7] sind hier einig, dass keine Terminierung der Surveillance bzw. des Follow-ups sinnvoll ist. Inwieweit bei Patienten, die sich aus medizinischen Gründen keinerlei operativem Eingriff unterziehen können, auf die Surveillance verzichtet werden kann, ist noch in der Diskussion. In der Praxis umfasst die Überwachung eine jährliche Bildgebung, bevorzugt mit MRT/MRCP. Hier gibt es erste Erfahrungen mit einem Kurzprotokoll für Kontrollen, das z. B. auf die Gabe von Kontrastmittel verzichtet [21]. Die Endosonographie kann alternativ im Wechsel erfolgen, daneben kann CA 199 zur Verlaufskontrolle sinnvoll sein [3]. Bei pankreasspezifischen Beschwerden oder anderen neu aufgetretenen Symptomen (z. B. Gewichtsverlust) sollte eine 
Bildgebung auch außerhalb der vorgegebenen Intervalle durchgeführt werden. Ohne die Patienten zu beunruhigen, sollten sie darauf hingewiesen werden, sich bei Symptomen unmittelbar zu melden. Tatsächlich ist der psychologische Stress der Surveillance für diese Gruppe von Individuen und Patienten gering [15] mit der Ausnahme von Individuen, deren Angehörige jung ein Pankreaskarzinom entwickelt haben [14].

Ein weiteres Screening von IPMNPatienten hinsichtlich Kolonadenome oder Barrett-Veränderungen wurde in der Vergangenheit aufgrund einer möglicherweise erhöhten Inzidenz dieser Erkrankungen bei IPMN-Patienten empfohlen; nach neuerer Datenlage scheint diese Assoziation jedoch nicht hinreichend belegt $\mathrm{zu}$ sein, um eine speziell strukturierte Überwachung außerhalb der grundsätzlichen Empfehlungen (z. B. zur Vorsorgekoloskopie) zu empfehlen.

\section{Fazit für die Praxis}

\section{- Fehlende Langzeitstudien sowie die komplexe Biologie der zystischen Pankreastumoren legen eine in- terdisziplinäre Vorgehensweise bei diesen Patienten nahe, die neben Gastroenterologen und Chirurgen erfahrene Radiologen, Pathologen (Zytologen) sowie interventionelle Endoskopiker (ERCP mit Spy Glass ${ }^{\mathrm{Tm}}$ DS/pCLE; EUS) einschließen sollte. \\ - Entscheidungen sollten idealerweise im Rahmen einer multidisziplinären Teamkonferenz getroffen werden.}

\section{Korrespondenzadresse}

\section{Prof. Dr. J.-M. Löhr}

Gastrozentrum, Karolinska University Hospital und Karolinska Institutet

Hälsovägen, SE-141 86 Stockholm, Schweden matthias.lohr@ki.se

\section{Einhaltung ethischer Richtlinien}

Interessenkonflikt. J.-M. Löhr und T. Hackert geben an, dass kein Interessenkonflikt besteht.

Dieser Beitrag beinhaltet keine von den Autoren durchgeführten Studien an Menschen oder Tieren.
Open Access. Dieser Artikel wird unter der Creative Commons Namensnennung 4.0 International Lizenz (http://creativecommons.org/licenses/by/4.0/deed. de) veröffentlicht, welche die Nutzung, Vervielfältigung, Bearbeitung, Verbreitung und Wiedergabe in jeglichem Medium und Format erlaubt, sofern Sie den/die ursprünglichen Autor(en) und die Quelle ordnungsgemäßnennen, einen Linkzur Creative Commons Lizenz beifügen und angeben, ob Änderungen vorgenommen wurden.

\section{Literatur}

1. Abdeljawad K, Vemulapalli KC, Schmidt CM et al (2014) Prevalence of malignancy in patients with pure main duct intraductal papillary mucinous neoplasms. Gastrointest Endosc 79:623-629

2. Arnelo U, Siiki A, Swahn F et al (2014) Singleoperator pancreatoscopy is helpful in the evaluation of suspected intraductal papillary mucinous neoplasms (IPMN). Pancreatology 14:510-514

3. BergerAW, Seufferlein T, KlegerA(2017)Cysticpancreatic tumors: diagnostics and new biomarkers. Chirurg 88:905-912

4. Campbell F, Verbeke CS (2013) Pathology of the pancreas. A practical approach. Springer, London

5. Canto MI, Almario JA, Schulick RD et al (2018) Risk of neoplastic progression in individuals at high risk for pancreatic cancer undergoing long-term surveillance. Gastroenterology 155(3):740-751.e2

6. Del Chiaro M, Verbeke CS, Kartalis N et al (2015) Short-term results of a magnetic resonance imaging-based Swedish screening program for individuals at risk for pancreatic cancer. JAMA Surg 150(6):512

7. European Study Group on Cystic Tumours of the Pancreas (2018) European evidence-based guidelines on pancreatic cystic neoplasms. Gut 67(5):789-804

8. Fritz S, Klauss M, Bergmann F et al (2012) Small (Sendai negative) branch-duct IPMNs: not harmless. Ann Surg 256:313-320

9. Hackert T, Fritz S, Klauss M et al (2015) Main-duct Intraductal papillary mucinous neoplasm: high cancer risk in duct diameter of 5 to $9 \mathrm{~mm}$. Ann Surg 262:875-880 (discussion 880-871)

10. Jang JY, Kim SW, Lee SE et al (2008) Treatment guidelines for branch duct type intraductal papillary mucinous neoplasms of the pancreas: when can we operate or observe? Ann Surg Oncol 15:199-205

11. Jang JY, Park T, Lee $S$ et al (2014) Validation of international consensus guidelines for the resection of branch duct-type intraductal papillary mucinous neoplasms. Br J Surg 101:686-692

12. Kaiser J, Fritz S, Klauss M et al (2017) Enucleation: a treatment alternative for branch duct intraductal papillary mucinous neoplasms. Surgery 161:602-610

13. Kim YI, Shin SH, Song KB et al (2015) Branch duct intraductal papillary mucinous neoplasm of the pancreas: single-center experience with 324 patients who underwent surgical resection. Korean J Hepatobiliary Pancreat Surg 19:113-120

14. Konings IC, Harinck F, Kuenen MA et al (2017) Factors associated with cancer worries in individuals participating in annual pancreatic cancer surveillance. Fam Cancer 16:143-151

15. Konings IC, Sidharta GN, Harinck F et al (2016) Repeated participation in pancreatic cancer surveillance by high-risk individuals imposes low psychological burden. Psychooncology 25:971-978
16. Löhr JM, Lonnebro R, Stigliano S et al (2015) Outcome of probe-based confocal laser endomicroscopy ( $p C L E$ ) during endoscopic retrograde cholangiopancreatography: a single-center prospective study in 45 patients. United European Gastroenterol J 3:551-560

17. Longnecker D, Hruban CA, Adler G et al (2010) Intraductal papillary-mucinous neoplasms of the pancreas. In: Hamilton SR, Aaltonen LA (Hrsg) Pathology and genetics of tumours of the digestive system. International Agency for Research on Cancer (IARC), Lyon

18. Madzak A, Olesen SS, Wathle GK et al (2016) Secretin stimulated MRI assessment of the benign pancreatic disorders systematic review and proposal for a standardized protocol. Pancreas 45:1092-1103

19. Megibow AJ, Baker ME, Morgan DE et al (2017) Management of incidental pancreatic cysts: a white paper of the ACR incidental findings committee. J Am Coll Radiol 14:911-923

20. Pandey P, Pandey A, Varzaneh FN et al (2018) Are pancreatic IPMN volumes measured on MRI images more reproducible than diameters? An assessment in a large single-institution cohort. Eur Radiol 28:2790-2800

21. Pozzi-Mucelli RM, Rinta-Kiikka I, Wünsche $K$ et al (2016) Pancreatic MRI for the surveillance of cystic neoplasms: comparison of a short with a comprehensive imaging protocol. Eur Radiol 27(1):41-50

22. Sahora K, Mino-Kenudson M, Brugge $W$ et al (2013) Branch duct intraductal papillary mucinous neoplasms: does cyst size change the tip of the scale? A critical analysis of the revised international consensus guidelines in a large single-institutional series. Ann Surg 258:466-475

23. Schmidt CM, White PB, Waters JA et al (2007) Intraductal papillary mucinous neoplasms: predictors of malignant and invasive pathology. Ann Surg 246:644-651 (discussion 651-644)

24. Seo N, Byun JH, Kim JH et al (2016) Validation of the 2012 international consensus guidelines using computed tomography and magnetic resonance imaging: branch duct and main duct intraductal papillary mucinous neoplasms of the pancreas. Ann Surg 263:557-564

25. Song KB, Kim SC, Park JB et al (2011) Singlecenter experience of laparoscopic left pancreatic resection in 359 consecutive patients: changing the surgical paradigm of left pancreatic resection. Surg Endosc 25:3364-3372

26. Tanaka M (2014) Thirty years of experience with intraductal papillary mucinous neoplasm of the pancreas: from discovery to international consensus. Digestion 90:265-272

27. Tanaka M, Fernandez-Del Castillo C, Kamisawa T et al (2017) Revisions of international consensus Fukuoka guidelines for the management of IPMN of the pancreas. Pancreatology 17:738-753

28. Vege SS, Ziring B, Jain R et al (2015) American gastroenterological association institute guideline on the diagnosis and management of asymptomatic neoplastic pancreatic cysts. Gastroenterology 148:819-822

29. Wong J, Weber J, Centeno BA et al (2013) High-grade dysplasia and adenocarcinoma are frequent in side-branch intraductal papillary mucinous neoplasm measuring less than $3 \mathrm{~cm}$ on endoscopic ultrasound. J Gastrointest Surg 17:78-84 (discussion p 84-75) 